\section{Putting cancer to REST}

\section{By Tim Fulmer, Staff Writer}

Although previous work has shown that either increasing or decreasing the level of repressor element-1-silencing transcription factor can induce tumors, it was unclear how to go about targeting or modulating the zincfinger DNA-binding protein. Two papers published in Nature separately describe the discovery of an E3 ubiquitin ligase that mediates the degradation of the transcription factor in a variety of cell types. Thus, targeting the ligase might be one strategy for modulating cellular levels of repressor element-1-silencing transcription factor and treating cancer.

Repressor element-1-silencing transcription factor (REST) has many functions: it is a transcriptional repressor in normal neuronal development, ${ }^{1}$ an oncogene when overexpressed in neural stem cells ${ }^{2}$ and a tumor suppressor in epithelial cells. ${ }^{3}$ Thus, excessively high or low levels of the protein's expression can result in cancer, depending on the cell type.

In the Nature papers, two independent research groups led by investigators at Harvard Medical School and New York University School of Medicine report that REST is ubiquitinated by the $\mathrm{SCF}^{\beta-\mathrm{TRCP}} \mathrm{E} 3$ ubiquitin ligase and targeted for proteasomal degradation in a variety of cultured cell lines. Both groups also found that prior phosphorylation of REST by an as yet unidentified kinase is required for REST ubiquitination ${ }^{4,5}$ (see Figure 1, "Regulation of intracellular repressor element-1-silencing transcription factor").

The Harvard group, led by Stephen Elledge and J. Wade Harper, showed that $\beta$-TRCP-mediated degradation of REST led to the differentiation of neural stem cells. The researchers also found that in human mammary epithelial cells, overexpression of $\beta$-TRCP lowered REST levels, which in turn led to oncogenic transformation.

Elledge is a professor of genetics at Harvard Medical School and at Brigham and Women's Hospital. Harper is a professor of pathology at Harvard Medical School.

The NYU group, led by Michele Pagano, a professor of oncology, showed that in multiple cell types, $\beta$-TRCP-mediated degradation of REST occurred during the G2 phase of the cell cycle. Low REST levels led to activation of the transcription of $\mathrm{Mad} 2$, a key protein that activates the mitotic spindle checkpoint and ensures proper alignment of chromosomes prior to completion of mitosis.
The group went on to show that a degradation-resistant REST mutant caused abnormally high REST levels in G2, which in turn induced multiple mitotic defects and subsequent chromosomal instability. That mechanism, the authors said, can contribute to tumor development.

\section{Throwing in a spanner}

Now that the molecular machinery controlling REST has been elucidated, the challenge is to disrupt the process.

Harper told SciBX that one strategy for preventing $\beta$-TRCP-mediated degradation of REST and subsequent oncogenic transformation would be to inhibit interactions between $\beta$-TRCP and the REST phosphodegron.

The phosphodegron is a region of the protein that comprises the phosphorylated amino acid side chains of two serine residues (S1027 and S1030) and surrounding amino acids.

"The REST phosphodegron may have a unique structural interaction with $\beta$-TRCP that could be targeted," said Harper. Key next steps, he said, would include obtaining a crystal structure of the REST phosphodegron bound to $\beta$-TRCP, carrying out high throughput enzymatic and binding assays and developing libraries of compounds for screening.

Don Payan, EVP and president of R\&D at Rigel Pharmaceuticals Inc., told $S c i B X$ that a fundamental challenge in designing inhibitors of the $\beta$-TRCP-REST interaction is that compounds would have to disrupt "a protein-protein interaction, not a single enzyme-substrate interaction. The latter has been the modeling paradigm for programs that develop inhibitors of kinases and proteases, for example. Unfortunately this paradigm has limited usefulness in the design of E3 ligase inhibitors."

Despite the challenge, Rigel has preclinical small molecule inhibitors that target three different E3 ligase-protein interactions: SCF-Skp2/p27 to treat cancer, Vif-BC-Cul5/APOBEC3G to prevent HIV infection and SCF-atrogin-1/calcineurin to treat muscle wasting diseases.

"Rigel's initial strategy of using high throughput enzymatic assays to isolate E3 ligase inhibitors foundered on difficulties in identifying where the inhibitors acted in a very complicated multisubunit complex," said Payan. "Consequently, our more recent attempts to discover and design E3 ligase inhibitors have combined assays with computational modeling and X-ray crystal structure data, where available."

Millennium Pharmaceuticals Inc. is taking a different approach to targeting E3 ligases: deactivating them before they have a chance to bind and ubiquitinate their substrate proteins.

"Rather than targeting the $\beta$-TRCP-REST interaction, our strategy is to go upstream from that event and target enzymes that activate the SCF ${ }^{\beta-T R C P}$ ligase in the first place," said CSO Joe Bolen. "One such enzyme is the Nedd8-activating enzyme, which transfers the Nedd8 protein to the cullin subunit of the E3 ligase, bringing about a conformational change that activates the ligase complex. It stands to reason 


\section{TARGETS \& MECHANISMS}

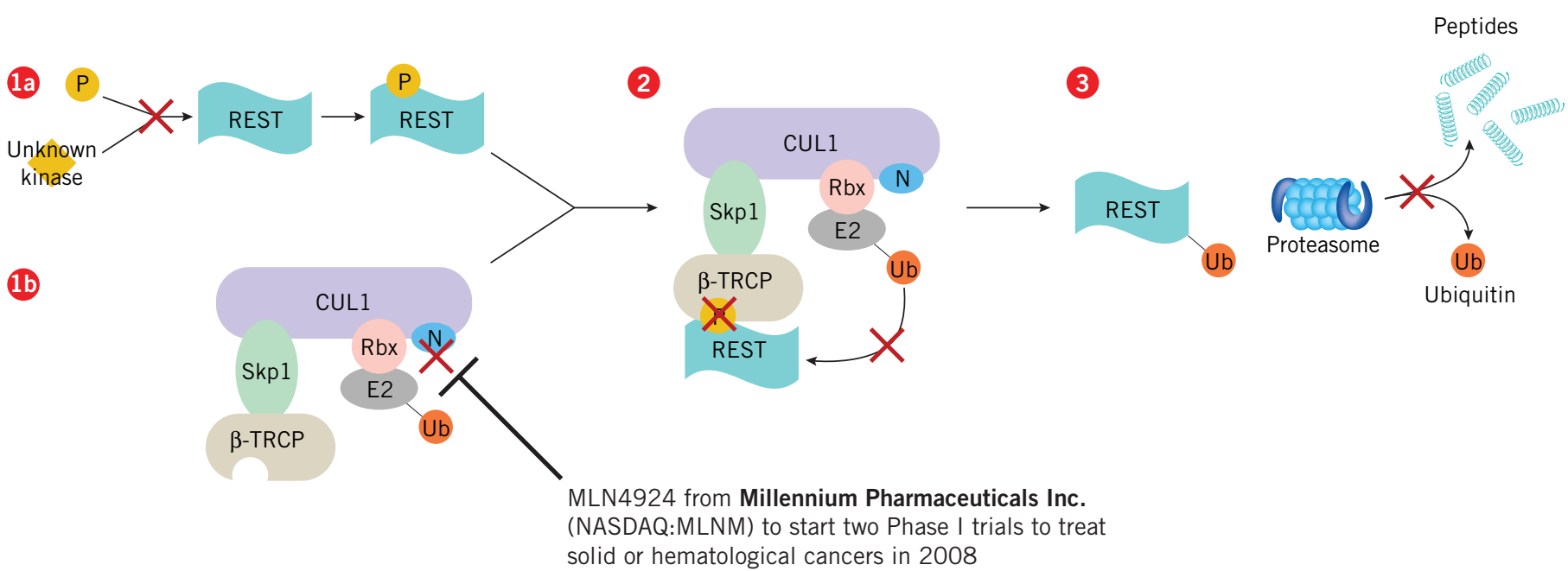

Figure 1. Regulation of intracellular repressor element-1-silencing transcription factor. Two Nature papers help unravel the machinery responsible for degrading repressor element-1-silencing transcription factor (REST), a potential cancer target, and reveal multiple therapeutic intervention points (marked by an X). Any therapeutic will need to be selective, however, as abnormal levels of REST can either stave off or lead to cancer depending on the type of cell. [1a] REST is initially phosphorylated by an as yet unidentified kinase, and blocking that phosphorylation would prevent the SCF ${ }^{\beta-T R C P}$ ligase from recognizing REST, thus saving it from downstream proteasomal degradation. [1b] The activated SCF ${ }^{\beta-T R C P}$ ligase is composed of the cullin 1 (Cul1) scaffold protein, to which S phase kinase-associated protein 1 (Skp1) and RING H2 finger protein $(\mathrm{Rbx})$ are bound. Skp1 binds the F-box protein $\beta$-TRCP. Nedd8 $(\mathrm{N})$ bound to Cul1 is required for activity of the complex; at least one biotech company, Millennium Pharmaceuticals Inc., is developing a compound to block Nedd8. [2] $\beta$-TRCP recognizes phosphorylated REST, triggering the transfer of ubiquitin (Ub) from Ub-conjugating enzyme (E2) to REST. Blocking Ub transfer would disrupt targeting of REST to the proteasome. [3] Finally, ubiquitinated REST is targeted to the $20 \mathrm{~S}$ proteasome, where it undergoes proteolysis. If that process were inhibited, cell-cycle arrest and apoptosis of cancer cells could occur. ${ }^{9}$

that inhibiting the neddylation step would deactivate the ligase and prevent it from ubiquitinating proteins downstream."

Added Bolen, "Designing small molecule inhibitors of an enzyme is easier than designing inhibitors of a protein-protein interaction."

Millennium's Nedd8-activating enzyme inhibitor MLN4924 has shown efficacy in preclinical cancer models.

"We've seen in tumors that MLN4924 deactivates cullin-based E3 ligases with subsequent accumulation of ligase client proteins within the cell and, ultimately, apoptosis," said Bolen. "Importantly, because the Nedd8-activating enzyme is more highly expressed in cancer cells than in normal cells, inhibiting its function has a much less toxic effect on noncancer cells."

This year, MLN4924 is slated to enter two Phase I trials to treat solid or hematological cancers.

Bolen noted that the E3 ligase described in

"Because REST shows
oncogenic or tumor
suppressor effects based
on cell type, REST-specific
ligase inhibitors for
therapeutic purposes would
probably also need to be
cell- or tumor-specific."
-Sadhan Majumder, M.D.
Anderson Cancer Center

ubiquitin-like post-translational modification pathways that could be targeted in cancer."

A third approach to targeting cellular REST levels would be to inhibit the unknown kinase that phosphorylates REST. Pagano told SciBX that next steps in his lab include studies to identify the kinase. the two Nature papers is a cullin-dependent ligase. "Since MLN4924 is designed to specifically target just such a ligase, there's every reason to believe the compound could effectively modulate REST levels in some cancer cell types," he said.

In January, Bolen added, Millennium announced a collaboration with Harper's group at Harvard to "identify components within various

\section{Getting at the cancer}

Given REST's different functions across a number of cell types, targeting the protein to treat a particular cancer could be a delicate task.

Sadhan Majumder of the University of Texas M.D. Anderson Cancer Center told SciBX that "because REST shows oncogenic or tumor suppressor effects based on cell type, REST-specific ligase inhibitors for therapeutic purposes would probably also need to be cellor tumor-specific."

Majumder, a professor of cancer genetics and neuro-oncology, is focusing on the dual role REST plays as a tumor suppressor in neurogenesis and as an oncogene in medulloblastomas. ${ }^{6,7}$

Ian Wood, professor of biological sciences at the University of Leeds, is studying how REST and its co-repressors recruit chromatin-modifying enzymes that subsequently affect gene regulation. ${ }^{8}$ "Experiments 


\section{TARGETS \& MECHANISMS}

that show tumor cells are more sensitive to loss of E3 ligase activity than normal cells-for example by using siRNA knockdown-would provide some encouragement that this could be a useful therapy," he said.

Raymond Deshaies, professor of biology at the California Institute of Technology, said one approach to studying the effects of tumorspecific targeting of E3 ligases would be to use "a mouse cancer model wherein the activity of the ligase could be regulated in some way in tumor cells-for example, by manipulating the expression of a knockedin allele with tetracycline or using conditional knockout of a specific allele."

Deshaies' research focuses on the mechanisms and regulation of ubiquitin-dependent proteolysis and cell division.

Pagano is also interested in performing a mutagenesis analysis of REST in human brain tumors.

"We expect to find mutations in the phosphor-motif of REST, as already observed for $\beta$-catenin, another oncogenic substrate of $\beta$ TRCP," he said. "These mutations would induce stabilization of REST and, consequently, chromosomal instability, thereby contributing to cell transformation."

Indeed, Majumder and colleagues previously found that many human medulloblastomas have higher levels of both REST and the myc oncogene than noncancerous tissue. ${ }^{2}$

\section{REFERENCES}

1. Ballas, N. et al. Cell 121, 645-657 (2005)

2. Su, X. et al. Mol. Cell. Biol. 26, 1666-1678 (2006)

3. Westbrook, T. et al. Cell 121, 837-848 (2005)

4. Westbrook, T. et al. Nature; published online March 20, 2008; doi:10.1038/nature06780

Contact: Stephen J. Elledge, Harvard Medical School, Boston, Mass. e-mail: selledge@genetics.med.harvard.edu

Contact: J. Wade Harper, Harvard Medical School, Boston, Mass. e-mail: wade_harper@hms.harvard.edu

5. Guardavaccaro, D. et al. Nature; published online March 20, 2008; doi:10.1038/nature06641

Contact: Michele Pagano, New York University School of Medicine, New York, N.Y.

e-mail: michele.pagano@nyumc.org

6. Majumder, S. Cell Cycle 5, 1929-1935 (2006)

7. Singh, S. et al. Nature; published online March 23, 2008; doi:10.1038/nature06863

8. Ooi, L. \& Wood, I. Nat. Rev. Genet. 8, 544-554 (2007)

9. Bonzini, P. et al. Leukemia 21, 838-842 (2007)

COMPANIES AND INSTITUTIONS MENTIONED

Brigham and Women's Hospital, Boston, Mass.

California Institute of Technology, Pasadena, Calif.

Harvard Medical School, Boston, Mass.

Millennium Pharmaceuticals Inc. (NASDAQ:MLNM), Cambridge, Mass.

New York University School of Medicine, New York, N.Y.

Rigel Pharmaceuticals Inc. (NASDAQ:RIGL), South San Francisco, Calif.

University of Leeds, Leeds, U.K.

University of Texas M.D. Anderson Cancer Center, Houston, Texas 